\title{
Perception des risques sanitaires dans le maraîchage à Abidjan, Côte d'Ivoire
}

\author{
Affou Séraphin WOGNIN ${ }^{1,2}$, Sebastien Koffi OUFFOUE ${ }^{2}$, \\ Emma Fernande ASSEMAND ${ }^{3}$, Kablan TANO ${ }^{1}$ et Rose KOFFI-NEVRY ${ }^{1 *}$ \\ ${ }^{1}$ Laboratoire de Biotechnologie et Microbiologie Alimentaire, Département des Sciences Alimentaires, \\ Université d'Abobo Adjamé, 02 BP 801 Abidjan 02, Côte d'Ivoire. \\ ${ }^{2}$ Laboratoire Central de l'Environnement du Centre Ivoirien Anti-pollution 20 BP 650 Abidjan 20, \\ Côte d'Ivoire. \\ ${ }^{3}$ Laboratoire de Biochimie et Technologie des Produits Tropicaux, Département des Sciences Alimentaires, \\ Université d'Abobo Adjamé, 02 BP 801 Abidjan 02, Côte d'Ivoire. \\ "Auteur correspondant; E-mail : rosenevry2002@yahoo.fr
}

\section{RESUME}

Cette étude a été conduite pour évaluer le niveau de connaissance et de perception des risques sanitaires dans le secteur du maraîchage à Abidjan, à travers une enquête transversale. Le marâichage est pratiqué à $77,98 \%$ par les hommes et à 22,02\% par les femmes, qui sont pour la plupart des analphabètes $(58,6 \%)$. Différents types de légumes sont produits à savoir les laitues $(40,97 \%)$, laitues+autres légumes $(32,12 \%)$ et autres légumes (26,9\%). 73,4\% des producteurs ne sont pas conscients des risques de contamination dus à leurs comportements contre $8,3 \%$ qui affirment reconnaître leur part de responsabilités dans la contamination des produits maraîchers. De manière préférentielle, $89,2 \%$ des exploitants agricoles utilisent des sacs en fil de coton pour le conditionnement des légumes frais qui sont transportés à $71 \%$ vers les points de vente au moyen de véhicules. En l'absence d'une éducation sanitaire fondée et des interventions localement adaptées, les cultivateurs n'accordent pas une attention particulière à la prévention des risques sanitaires.

(C) 2013 International Formulae Group. All rights reserved.

Mots clés: Conception, fruits et légumes, contamination, maraîchage périurbain, comportement des maraîchers.

\section{INTRODUCTION}

En Côte d'Ivoire, l'agriculture urbaine et périurbaine est pratiquée le plus souvent sur des sites marécageux. Ainsi, l'on assiste à un développement de la culture des légumes non loin des berges lagunaires et autres points marécageux situés à la périphérie de la ville. Les avantages sont l'approvisionnement rapide en produits frais (laitue, concombre, épinard, etc.) des populations urbaines (KoffiNevry et al., 2011). La consommation des fruits et légumes est recommandée dans plusieurs pays pour la protection contre des maladies telles que le cancer, l'obésité, les maladies cardio-vasculaires et les bienfaits de leurs fibres sont aussi reconnus dans le bon déroulement du transit intestinal (Berger et al., 2010 ; Idogun et al., 2008). L'un des légumes 
le plus apprécié et consommé au monde est la laitue (Lactuca sativa) qui est bien entrée dans les habitudes gastronomiques des Abidjanais (Côte d'ivoire) (Koffi-Nevry et al., 2011, 2012).

Les spéculations cultivées par les maraîchers d'Abidjan sont diversifiées (la tomate, le piment, la laitue, etc.) et la plupart des cultures boucle deux à trois cycles par an. Ainsi, les agriculteurs se concentrent sur la production de celles qui ont une faible durée de rotation (exemple, la laitue), elles s'écoulent assez facilement et sont beaucoup appréciés par les abidjanais (Kouakou, 2009). Cependant, les risques sanitaires associés aux conditions de production sont nombreux: eaux d'irrigation proviennent des réceptacles d'effluents urbains, pratiques polluantes ou peu hygiéniques de certains maraîchers etc.

La présente étude s'est proposé de cerner la réalité des risques sanitaires en agriculture urbaine de façon générale, et particulièrement ceux liées à la laitue (Lactuca sativa), à travers une démarche d'entretiens auprès des exploitants maraîchers. Les différentes informations recueillies vont permettre d'établir une base de données relative à la pratique du maraîchage dans le district d'Abidjan. A cet effet, il importe de connaître les caractéristiques des maraîchers et de cerner l'environnement de culture des légumes, en particulier celui de la laitue en agriculture périurbaine.

\section{MATERIEL ET METHODES}

\section{Zone d'étude}

Une prospection menée sur les différents sites de production à Abidjan a permis d'identifier trois principaux sites de production maraîchère sur lesquels a porté cette étude. Le site 1, d'une superficie d'environ 30 ha et dominé par la culture de la laitue, est situé dans la commune de Port bouet, proche de l'Aéroport Felix Houphouët Boigny. Le site 2, d'une superficie de 35 ha est situé dans la même commune au niveau du village Adjahui. Le site 3, avec une superficie de 23 ha est situé dans la commune de Cocody (M'pouto) en bordure de la lagune Ebrié (Figure 1).

\section{Enquête préliminaire}

La population d'étude est constituée d'exploitants maraîchers. Un questionnaire a été élaboré et divisé en deux sections: la première section a concerné l'âge, le sexe et le niveau d'étude des exploitants agricoles. La deuxième section était relative aux caractéristiques de production maraîchère. Cette enquête qui a duré dix mois a porté sur un total de 436 maraîchers repartis comme suit : 125 maraîchers pour le site 1,174 pour site 2 , et 137 du site 3 .

\section{Analyse statistique}

Les données qualitatives notamment celles issues des groupes focaux ont été interprété sous formes d'analyse croisée entre les stations. Les graphiques ont été obtenus grâce au logiciel Excel (version 2007). L'analyse de variance à un facteur (ANOVA) a été effectuée avec le logiciel Graphpad Prism 5.0 pour la comparaison des paramètres (variables réponses) étudiés. Pour les différences significatives entre ces paramètres (variables réponses), le classement des moyennes a été fait selon le test de Neumannkeuls au seuil 5\%. Les différences sont considérées comme significatives pour des valeurs de $\mathrm{P}<0,05$. 


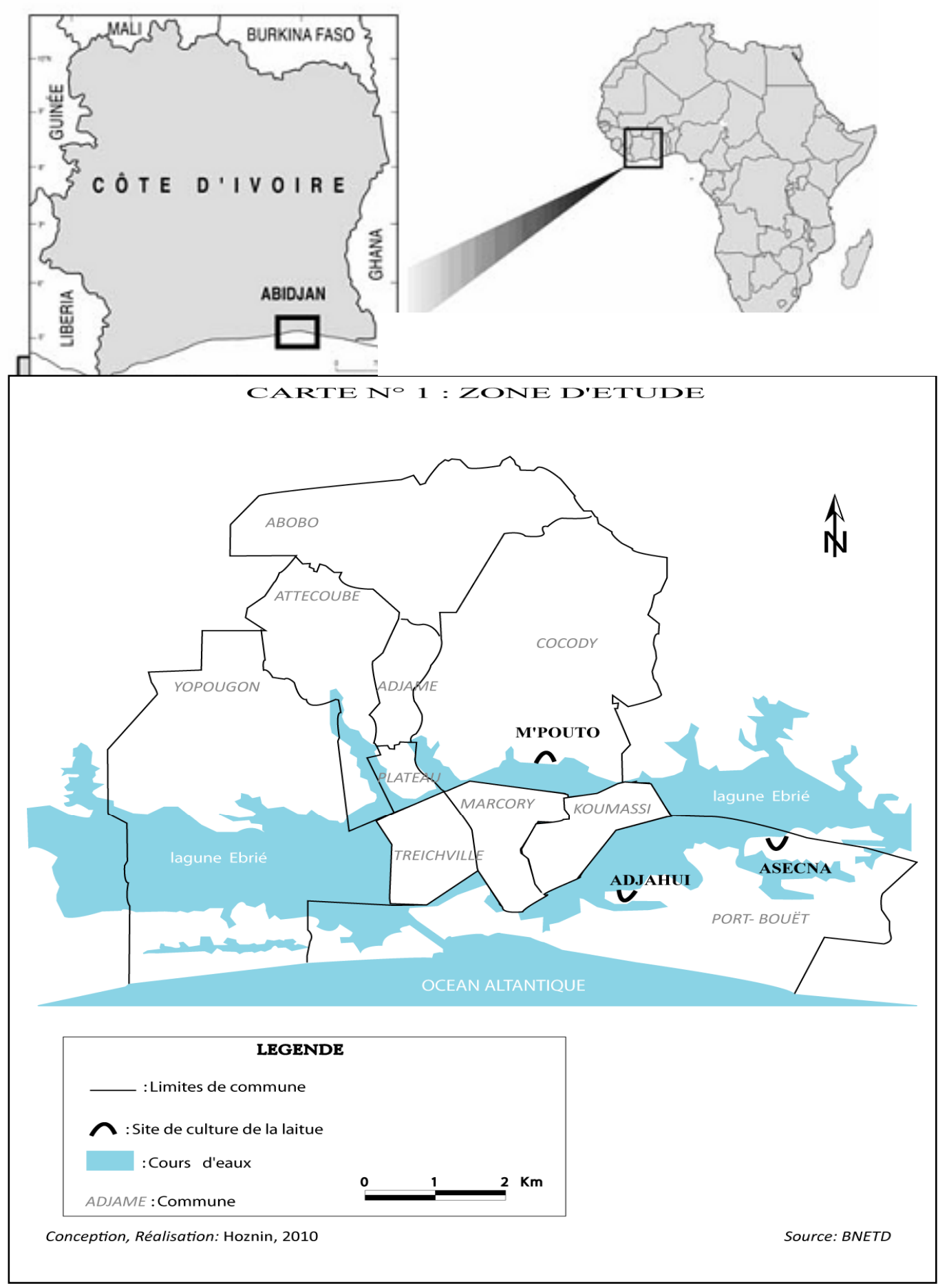

Figure 1 : Photographie de la carte de la ville d'Abidjan présentant les différentes communes et les sites de production maraîchère. 


\section{RESULTATS}

$\mathrm{La}$ répartition des légumes est présentée dans le Tableau 1. Les cultures produites sont des légumes-feuilles (laitue, épinard et menthe), des légumes-fruits (gombo, aubergines, piment, courgette, concombre et poivron) et des légumes-bulbes (oignon, ail et poireau). Environ 67\% des répondants utilisent les céanes comme sources d'eau pour l'arrosage des légumes contre $26 \%$ pour les puisards, et $7 \%$ pour l'eau naturelle. Seulement $1,64 \%$ des producteurs utilisent l'eau de la lagune Ebrié pour l'irrigation de leurs parcelles (Figure 2). Le Tableau 2 indique que le maraîchage est pratiqué majoritairement par des hommes $(77,98 \%)$ contre seuleument $22,02 \%$ de femmes. Il est dominé à $68,6 \%$ par des jeunes dont l'âge varie de 15 à 35 ans; les adultes de plus de 35 ans représentent $31,4 \%$. Les maraîchers sont à $58,6 \%$ des analphabètes. Toutefois, $41,4 \%$ des producteurs prétendent avoir été scolarisés avec un niveau d'étude très faible.

Le Tableau 3 révèle que $48,40 \%$ des maraîchers du site 1 exploitent uniquement la laitue contre $30,50 \%$ de producteurs qui pratiquent à la fois la culture de la laitue et celle d'autres légumes. Sur le site 2, 19,18\% des exploitants cultivent la laitue contre 43,3 $\%$ qui produisent autres légumes que la laitue. Il existe une différence significative $(\mathrm{P}<0,05)$ entre les maraîchers qui produisent uniquement la laitue et ceux qui cultivent d'autres légumes. Par ailleurs, $81,3 \%$ des maraîchers du site 1 ont attesté ne pas connaître les risques liés aux pratiques maraîchères contre $64,94 \%$ pour le site 2 , et $73,97 \%$ pour le site 1 . D'un site à l'autre, aucune différence significative $(P>0,05)$ n'a été observée pour la reconnaissance et l'ignorance des risques de contamination des légumes par les exploitants maraîchers. Les infections de légumes ont été attribuées à 69,86\% aux microorganismes du sol par les producteurs du site 3 , à $66,4 \%$ aux arthropodes par ceux du site 2 et à la mauvaise qualité de l'eau d'arrosage des légumes par les maraîchers du site 1. Aucune différence significative n'a été observée entre les réponses des producteurs (Tableau 3). Il ressort du Tableau 4 que la majorité des maraîchers, soit plus de $90 \%$ quelque soit le site, utilise régulièrement les produits phytosanitaires dans la lutte contre les nuisibles. Des sacs en fil de coton préférentiellement utilisés pour le conditionnement des légumes, soit $92,8 \%$ pour le site $1,81,6 \%$ pour le site 2 , et $93,2 \%$ pour le $3^{\mathrm{e}}$ site. Les légumes frais après leur récolte sont transportés entre $65,6 \%$ et $76,7 \%$ des cas, vers les points de vente au moyen de véhicules contre $21,3 \%$ pour le site 2 qui transportent les légumes à pieds (Tableau 4) et $27,20 \%$ des exploitants du site 1 qui utilisent des charrettes à traction humaine appelé communément «pousse-pousse».

Tableau 1 : Nature des légumes cultivés sur les sites de production investigués.

\begin{tabular}{lccc}
\hline Sites production & Légumes-feuilles & Légumes cultivés & Légumes-bulbes \\
\cline { 2 - 4 } Site 1 & $\begin{array}{c}\text { Abondant dominé } \\
\text { par la laitue }\end{array}$ & $\begin{array}{c}\text { Peu abondant dominé : } \\
\text { aubergine, gombo }\end{array}$ & Absence \\
\hline Site 2 & $\begin{array}{c}\text { Peu abondant dominé } \\
\text { par la laitue }\end{array}$ & $\begin{array}{c}\text { Abondant : concombres, } \\
\text { aubergines, piment, gombo }\end{array}$ & Absence \\
\hline Site 3 & $\begin{array}{c}\text { Abondant dominé } \\
\text { par la laitue }\end{array}$ & $\begin{array}{c}\text { Peu abondant : aubergines, } \\
\text { piment }\end{array}$ & Absence \\
\hline
\end{tabular}


Tableau 2 : Répartition (\%) des caractéristiques des producteurs de légumes dans la zone péri-urbaine d'Abidjan.

\begin{tabular}{lcc}
\hline Caractéristiques & \multicolumn{2}{c}{ Répartition } \\
\cline { 2 - 3 } & Fréquence (effectif) & Pourcentage \% \\
\hline Age (années) & 110 & 25,2 \\
$15-25$ & 189 & 43,4 \\
$25-35$ & 93 & 21,3 \\
$35-45$ & 44 & 10,1 \\
$>45$ & 436 & 100 \\
Total & & \\
\hline Sexe & 340 & 77,98 \\
Hommes & 96 & 22,02 \\
Femmes & & \\
\hline Niveau d'étude & 256 & 58,6 \\
Non scolarisés & 123 & 28,3 \\
Primaire & 53 & 12,1 \\
$1^{\text {er }}$ cycle secondaire & 04 & 1 \\
$>3^{\text {ième }}$ & & \\
\hline
\end{tabular}

Tableau 3 : Sources et risques d'infection liés à la culture des légumes.

\begin{tabular}{|c|c|c|c|}
\hline & Site 1 & Site 2 & Site 3 \\
\hline \multicolumn{4}{|l|}{ Légumes cultivés } \\
\hline Laitue & $48,4 \pm 4,5 \mathbf{a}$ & $19,18 \pm 2,3 \mathbf{b}$ & $55,34 \pm 4,9 \mathbf{a}$ \\
\hline Laitue+autres & $30,50 \pm 3,3 \mathbf{b}$ & $37,52 \pm 4,18 \mathbf{a}$ & $28,35 \pm 2,8 \mathbf{b}$ \\
\hline Autres légumes & $21,10 \pm 2,9 \mathbf{b c}$ & $43,3 \pm 3,2 \mathbf{a}$ & $16,3 \pm 0,9$ bc \\
\hline \multicolumn{4}{|c|}{ Connaissance des risques } \\
\hline Connaissance & $6,4 \pm 2,33 \mathbf{a b}$ & $10,37 \pm 1,61 \mathbf{a c}$ & $8,20 \pm 4,18 \mathbf{a}$ \\
\hline Non connaissance & $12 \pm 2,9 \mathbf{b}$ & $24,72 \pm 4,74 \mathbf{a b}$ & $17,80 \pm 2,12 \mathbf{b}$ \\
\hline Ignorance & $81,3 \pm 7,53 \mathbf{a}$ & $64,94 \pm 6,19 \mathbf{a b}$ & $73,97 \pm 5,12 \mathbf{a}$ \\
\hline \multicolumn{4}{|c|}{ Sources d'infections } \\
\hline Arthropodes & $24 \pm 2,3 \mathbf{b}$ & $66,4 \pm 4 \mathbf{a}$ & $13,4 \pm 1,6 \mathbf{b c}$ \\
\hline Eau arrosage & $62,4 \pm 3,3 \mathbf{a}$ & $27,01 \pm 3 \mathbf{b}$ & $13,7 \pm 0,7 \mathbf{b c}$ \\
\hline Microbes du sol & $13,6 \pm 0,9 \mathbf{b c}$ & $6,99 \pm 3,5 \mathbf{c}$ & $69,86 \pm 9,9 \mathbf{a}$ \\
\hline
\end{tabular}


Tableau 4 : Modes de gestion des légumes cultivés.

\begin{tabular}{|c|c|c|c|}
\hline & Site 1 & Site 2 & Site 3 \\
\hline \multicolumn{4}{|c|}{ Traitement des légumes } \\
\hline - régulier & $94,4 \pm 4,4 \mathbf{a}$ & $91,38 \pm 4 \mathbf{a}$ & $94,52 \pm 4 \mathbf{a}$ \\
\hline - non régulier & $5,6 \pm 1,5 \mathbf{b}$ & $8,62 \pm 0,4 \mathbf{a b}$ & $5,48 \pm 0,4 \mathbf{b}$ \\
\hline - absence & $0 \mathbf{c}$ & $0 \mathbf{c}$ & $0 \mathbf{c}$ \\
\hline \multicolumn{4}{|c|}{ Mode de conditionnement } \\
\hline - sacs (fil coton) & $92,8 \pm 2,7 \mathbf{a}$ & $81,6 \pm 4 \mathbf{b}$ & $93,2 \pm 2,4 \mathbf{a}$ \\
\hline - cuvettes & $1,6 \pm 0,5 \mathbf{c}$ & $8,62 \pm 0,7 \mathbf{a b}$ & $5,48 \pm 0,7 \mathbf{b}$ \\
\hline - sachets & $5,6 \pm 0,4 \mathbf{b}$ & $9,77 \pm 0,5 \mathbf{a b}$ & $1,37 \pm 0,03 \mathbf{c}$ \\
\hline \multicolumn{4}{|c|}{ Mode de transport } \\
\hline - véhicule & $65,6 \pm 4,2 \mathbf{a}$ & $70,7 \pm 17,2 \mathbf{a}$ & $76,7 \pm 3,4 \mathbf{a}$ \\
\hline - marche & $7,2 \pm 0,8 \mathbf{c}$ & $21,3 \pm 0,9 \mathbf{a b}$ & $15,1 \pm 0,9 \mathbf{b}$ \\
\hline - charrette & $27,2 \pm 1,4 \mathbf{a b}$ & $8,04 \pm 1,2 \mathbf{b}$ & $8,2 \pm 0,9 \mathbf{b c}$ \\
\hline
\end{tabular}

En ligne et en colonne, les valeurs portant les mêmes lettres sont égales selon le test de Neumann-keuls $(P=0,05)$.

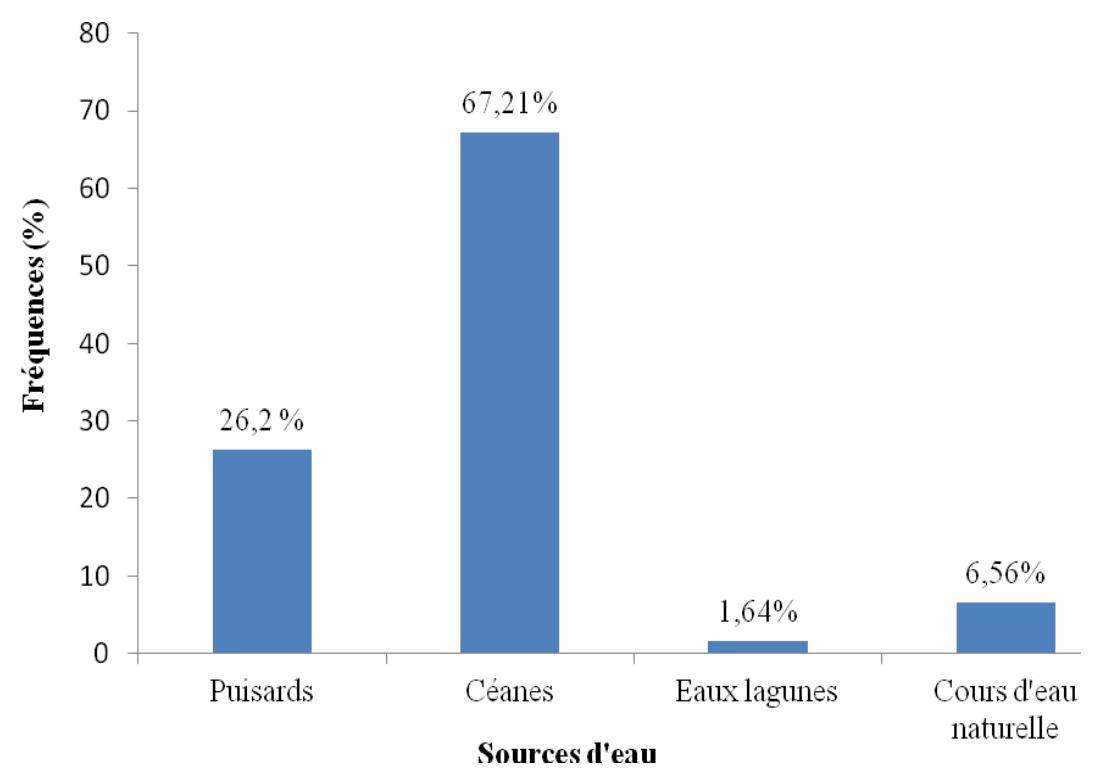

Figure 2 : Fréquence des différentes sources d'eau utilisées pour l'arrosage des légumes.

\section{DISCUSSION}

La pratique du maraîchage à Abidjan, participe à la lutte contre la pauvreté en rehaussant les sources de revenues des populations démunies vivant dans les quartiers précaires. Cette observation est en adéquation avec celle de plusieurs auteurs qui ont indiqué que l'agriculture urbaine participe dans un environnement urbain de façon intégré à la lutte contre la pauvreté et l'assainissement environnemental (Parrot, 2008; Conchita et al., 2010). Selon Compaoré et al. (2010), elle participerait à l'assainissement des villes en utilisant comme engrais, les déchets recyclés 
et les eaux usées traitées. Le maraîchage se développe en fait, pour des fonctions d'autosubsistance ou d'approvisionnement des marchés locaux. Le fait que le maraîchage soit dominé par la culture de la laitue pourrait s'expliquer en partie par le fait que le climat d'Abidjan est favorable aux exigences de culture de ce légume feuille (Kouakou, 2009). Les résultats de cette étude sur les eaux de céanes et les puisards qui sont les principales sources d'eaux les plus utilisés pour le marâichage à Abidjan sont en accord avec ceux de Adiko et al. (2010) qui ont montré que les puits traditionnels sont les sources d'eau les plus utilisées (51\%). La faible représentativité des femmes dans la production maraîchère pourrait être liée à la pénibilité des pratiques d'irrigation dominée par le travail manuel. Cette observation a été également faite par Kenmongue et al. (2010) qui ont montré que le maraîchage est pratiqué par $77 \%$ d'hommes contre $23 \%$ femmes dont la tranche d'âge est comprise entre 24 et 68 ans. La présence plus marquée des hommes pourrait s'expliquer par l'existence de groupements d'hommes dans le maraîchage à Abidjan. Le faible niveau d'instruction des exploitants maraîchers à Abidjan pourrait être dû au fait que le maraîchage est une activité qui n'exige pas de compétences particulières.

La nature informelle, le niveau élevé d'analphabétisme et l'absence de programmes de formation sur les bonnes pratiques en matière d'exploitation agricole urbaine pourraient justifier le comportement des producteurs qui ne sont pas conscients des risques de contamination dus aux pratiques du maraîchage. Ces résultats soutiennent ceux de Keraita et al. (2008) qui ont montré à travers des travaux réalisés à Accra (Ghana), que près de $80 \%$ des agriculteurs interrogés ne percevaient pas le risque sanitaire lié à l'utilisation des eaux usées pour l'arrosage des légumes. Cependant, ceux qui sont conscients des risques potentiels pour la santé publique, les sous estiment et privilégient les avantages économiques liés à l'utilisation d'eau polluée pour l'irrigation des légumes comme l'ont aussi constatés Jeroen et al. (2007). Les résultats de cette étude soutiennent ceux de Cissé (1997) à travers les idées et croyances des exploitants maraîchers à Ouagadougou. En effet, les maraîchers refusent d'accepter un lien possible entre la qualité de l'eau d'arrosage et certaines maladies, et donc une responsabilité dans la chaîne des risques sanitaires. Des résultats similaires ont été signalés dans d'autres études réalisées dans les communautés pauvres (Marenya et Barrett, 2007 ; Peres et al., 2006).

La minorité d'agriculteurs $(8,31 \%)$ est consciente des risques liés à l'eau d'arrosage et se disent impuissants face à ce système compte tenu du fait qu'ils travaillent en permanence dans un environnement physique dégradé dû aux dépôts d'ordures de tout genre, à l'immersion permanente dans l'eau et à l'utilisation de produits phytosanitaires dont ils ignorent les effets sur l'homme. Les résultats de cette étude sont en désaccord avec ceux d'une étude conduite par Kilelu (2004). Selon ce dernier les maraîchers de Nairobi (Kenya) et Kumasi (Ghana) sont plus préoccupés par la qualité de l'eau d'arrosage et de son potentiel à causer des maladies.

A Abidjan, les exploitants agricoles travaillent sur les sites de production dans les conditions d'extrême précarité sans le minimum d'équipement de protection nécessaire (gangs, bottes combinaisons...). Selon eux et nous citons : «Lorsqu'on soulève l'arrosoir, l'eau ruisselle sur tout le corps et même quand on porte des gangs et des bottes on n'y peut rien dans la mesure où le niveau de l'eau dans le puits dépasse les genoux». Ce constat confirme celui de Knudsen et al. (2008); Amoah et al. (2007) dans une étude réalisée au Ghana où seulement $19 \%$ des agriculteurs portaient des vêtements de protection, (bottes et gants) non en raison des risques pour la santé, mais pour se protéger contre les lésions dues au froid et aux blessures physiques. De telles observations ont également été faites par d'autre auteurs (Matthys et al., 2006). 
Pour lutter contre les problèmes sanitaires dus aux insectes nuisibles dans le maraîchage périurbain d'Abidjan, la majorité des exploitants agricoles $(93,4 \%)$ appliquent un traitement phytosanitaire régulier mais diversifiés et à des doses inappropriés ; ce qui pourrait constituer un risque sanitaire dans la production des légumes. Un autre facteur de risque sanitaire se situe après la récolte. En effet, au cours du transport, la mise en commun des légumes peut entraîner une contamination croisée des produits sains. En outre, les véhicules utilisés pour le transport des légumes vers les marchés de gros ne sont pas régulièrement nettoyés. Tout ceci augmente les risques liés à la consommation de ces produits.

\section{Conclusion}

Il ressort de cette étude que la culture des légumes est dominée par la laitue. Il existe une grande hétérogénéité dans les caractéristiques socio-économique des maraîchers. Les possibilités inquiétantes de propagation des maladies d'origine alimentaire existent donc dans le maraîchage à Abidjan. Une éducation sanitaire serait nécessaire pour prévenir les risques sanitaires liés à la pratique du maraîchage et éviter des épidémies éventuelles.

\section{REFERENCES}

Adiko A, Matthys B, Cissé G, Bonfoh B, Tanner M, Utzinger J. 2010. Relation entre le capital humain des maraîchers urbains et leurs comportements de prévention des risques sanitaires sur les sites de culture à Abidjan (Côte d'Ivoire). VertigO, 10(2): 1-9.

Amoah P, Drechsel P, Henseler M, Abaidoo RC. 2007. Irrigated urban vegetables production in Ghana: microbiological contamination in farms and markets and associated consumer risk groups. J. Water Health, 5(3): 455-466.

Berger CN, Sodha SV, Shaw RK, Griffin PM, Pink D, Hand P, Frankel G. 2010. Minireview: Fresh fruit and vegetables as vehicles for the transmission of human pathogens. Environ. Microbiol., 12: 23852397.

Cissé G. 1997. Impact sanitaire de l'utilisation d'eaux polluées en agriculture urbaine: cas du maraîchage à Ouagadougou (Burkina Faso). Thèse No 1639, Ecole Polytechnique Fédérale de Lausanne, p. 310.

Compaoré E, Nanema LS, Bonkoungou S, Sedogo MP. 2010. Évaluation de la qualité de composts de déchets urbains solides de la ville de Bobo-Dioulasso, Burkina Faso pour une utilisation efficiente en agriculture. Journal of Applied Biosciences, 33: 2076-2083.

Conchita MG, Kêdowidé M, Sedogo P, Cissé G. 2010. Dynamique spatio temporelle de l'agriculture urbaine à Ouagadougou: Cas du Maraîchage comme une activité montante de stratégie de survie. VertigO, 10(2). Online.

Idogun ES, Famodu AA, Olasunkanmi LA, Osilesi O, Adebawo OO. 2008. Effects of fruits and vegetables on electrolytes and blood pressure of hypertensive patients seen in Nigeria. African Journal of Food Agriculture and Nutrition Development, 8(3): 349-357.

Jeroen H, Ensink J, Mahmood T, Dalsgaard A. 2007. Wastewater-irrigated vegetables: market handling versus irrigation water quality. Tropical Medicine and International Health, 12(10): 1365-3156.

Kenmongue GR, Rosillon F, Mpakam HG, Nono A. 2010. Enjeux sanitaires, socioéconomiques et environnementaux liés à la réutilisation des eaux usées dans le maraîchage urbain : cas du bassin versant de l'Abiergué (Yaoundé-Cameroun), p. 25.

Keraita B, Drechsel P, Konradsen F. 2008. Perceptions of farmers on health risks and risk reduction measures in wastewaterirrigated urban vegetable farming in Ghana. Journal of Risk Research, 11(8): 1047-1061. 
Kilelu C. 2004. Wastewater irrigation and farmer's perception of health risks and institutional perspectives: A case study from Maili Saba, Nairobi, CFP Report 38, IDRC, Ottawa.

Knudsen L, Phuc P, Hiep N, Samuelson H, Jensen P, Dalsgaard A, Raschid-Sally L, Konradsen F. 2008. The fear of awful smell: risk perceptions among farmers in Vietnam using wastewater and human excreta in agriculture. Southeast Asian Journal of Tropical Medicine and Public Health, 39(2): 341-352.

Koffi-Nevry R, Assi-Clair BJ, Assemand EF, Wognin AS, Koussemon M. 2012. Origine des témoins de contamination fécale de l'eau d'arrosage de la laitue (lactuca sativa) cultivée dans la zone péri urbaine d'Abidjan. Journal of Applied Biosciences, 52: 3669-3675.

Koffi-Nevry R, Assi-Clair BJ, Koussémon M, Wognin AS, Coulibaly N. 2011. Potential enterobacteria risk factors associated with contamination of lettuce (Lactuca sativa) grown in the peri-urban area of Abidjan (Côte d'Ivoire). Int. J. Biol. Chem. Sci., 5(1): 279-290.

Kouakou KJ. 2009. Etude des métaux traces $(\mathrm{Cd}, \mathrm{Cu}, \mathrm{Pb}, \mathrm{Zn}, \mathrm{Ni})$ dans les sols et les produits maraîchers de deux sites d'agriculture dans la ville d'Abidjan (Côte d'Ivoire). Thèse de Doctorat
Unique de L'Université d'AboboAdjamé, Abidjan, Côted'Ivoire, p. 145.

Marenya P, Barrett C. 2007. Household-level determinants of adoption of improved natural resources management practices among smallholder farmers in western Kenya, Food Policy, 32: 515-536.

Matthys B, Adiko FA, Cissé G, Wyss K, Tschannen AB, Tanner M, Utzinger J. 2006. Le réseau social des maraîchers à Abidjan agit sur la perception des préoccupations et des risques sanitaires liés à l'eau. VertigO - la Revue Electronique en Sciences de l'Environnement, hors-série 3, décembre.

Parrot L. 2008. Agricultures et Développement Urbain en Afrique Subsaharienne: Gouvernance et Approvisionnement des Villes. Ed. L'Harmattan : p. 208.

Peres F, Moreira C, Rodrigues M, Claudio L. 2006. Risk perception and communication regarding pesticide use in rural work: A case study of Rio de Janeiro State, Brazil. International Journal of Occupational and Environmental Health, 12: 400-407. 